\title{
Spintronic Faraday rotation spectroscopy and geometrical modulation of spin current in an Aharonov-Casher ring
}

\author{
Zhongshui Ma, ${ }^{1,2,3}$ Peng Li, ${ }^{3}$ and Shun-Qing Shen ${ }^{3}$ \\ ${ }^{1}$ State Key Laboratory for Mesoscopic Physics and Department of Physics, Peking University, Beijing 100871, China \\ ${ }^{2}$ Advanced Research Center, Zhongshan University, Guangzhou 510275, China \\ ${ }^{3}$ Department of Physics, The University of Hong Kong, Pukfulam Road, Hong Kong, China
}

(Received 7 March 2004; published 24 September 2004)

\begin{abstract}
We study the ballistic spin transmission through an AC ring between two ferromagnetic filter-electrodes in the presence of spin-orbit interaction, theoretically. It is shown that the spin precession of polarized electrons can be controlled via a cylindrically symmetric electric field, and the sequence of the polarized conductance depends on the relative orientation of magnetization between the left and right filters. The injected spinpolarized electrons can be blocked completely for the special values of electric field and angle of texture, which makes the magnetoresistance to be drastically enhanced. We also propose an interference scheme to detect the geometric phase.
\end{abstract}

DOI: $10.1103 /$ PhysRevB.70.125318

PACS number(s): 73.23.-b, 73.40.Gk

Stimulated by the pioneering proposal of spin-transistor by Datta and Das, ${ }^{1}$ much effort has been spent on an efficient mechanism for achieving spin-polarized electron injection into semiconductors. The original idea came from the spinresolved conductance manifested itself as the modulation of spin current, which is produced by spin precession via the spin-orbit (SO) interaction or Rashba coupling when the spin carriers transport through a narrow-gap semiconductor. Because the strength of Rashba coupling depends on the external gate voltage, therefore, it evidently makes the spin modulation controllable. The studies have covered over from the semiclassical diffusive regime to the ballistic regime of spindependent transport. Presently, how to control and to utilize the well-known materials and well-controlled structures are intrigued more and more from both the experimental and theoretical sides, inspired by the promises of its application in magnetoelectric devices and quantum computation. Experimentally, the electric control of spin coherence ${ }^{2}$ and coherent spin precession during transport ${ }^{3}$ were studied by using the optical generation and detection techniques in semiconductors. Spin-valve transistor structures have been proposed. ${ }^{4}$ To achieve a high-spin polarization, various structures of semiconductor heterostructures, which relied on spin degeneracy of conduction electron band lifted by SO interaction or Rashba coupling, have been studied. In the multilayered configuration of nonmagnetic semiconductors, the nonmagnetic current was predicted to induce spin precession in various multilayers, which consist of the ferromagnetic layers. ${ }^{5}$ Quantum effects in novel spintronic devices, such as the quantum coherence and interference, have also been taken into account recently. ${ }^{6}$ However, most works in the investigation of spintronic devices are practically demonstrated with some kinds of semiconductor heterostructures. The extraneous effect, risen from a resistance mismatch between these quite different materials, makes the results controversial. Alternatively, based on the magnetic semiconductor, the spin-switch ${ }^{7}$ and spin-dependent filter ${ }^{8}$ effects have been demonstrated, theoretically. Spin-polarized current in ballistic mesoscopic rings is raised due to the Zeeman cou- pling in the presence of magnetic textures. It is shown that the modulation of the current was interpreted as a possible manifestation of Aharonov-Bohm effect. Therefore, the geometric modulation of spin-polarized current emerges its possibility in the control function of polarization. However, there is also practical difficulty in the experimental achievement to low temperature and in the uncertain controllable effect for the local inhomogeneous magnetic field. In a recent paper ${ }^{9}$ we have exploited this geometric analogy to show that the spin precession can be induced by the Aharonov-Casher (AC) effect. ${ }^{10}$ It is shown that the process of spin-resolved current in the incoming spin-polarized electron is emerged through the AC effect. In such an AC ring, the ballistic transport regime is more easily fulfilled experimentally and even at higher temperatures. The modulation originates from quantum spin interference with a specific phase difference risen by a geometric phase, i.e., Aharonov-Anandan (AA) phase, ${ }^{11}$ which is acquired by the spin carrier as it travels around the ring. We have shown a scheme of the geometrical control of spin precession, which differs from the common electrical control of spin precession in the semiconductor heterostructures. The preferentially particular spin electrons in the detectors are, therefore, modulated by the geometric phase. This has raised a hope for the realization of geometrically modulated semiconductor nonmagnetoelectronic devices. We believe geometric control of a spin degree of freedom in solid-state physics to have sound potential in the applications of quantum spintronic devices.

In this paper we study the geometric spintronic Faraday rotation spectroscopy by means of the geometrically controlled strength of polarization, which describes the complete polarization of electrons by means of spin precession in electron propagation direction in a special setup structure. A basic structure is nonmagnetic ballistic mesoscopic rings connected with two ferromagnetic filters as spin injector and detector. A textured electric field $\mathbf{E}$, generated by a perpendicular charged line through the ring, as well as a point charge near the center of the ring and a circular gate voltage, 


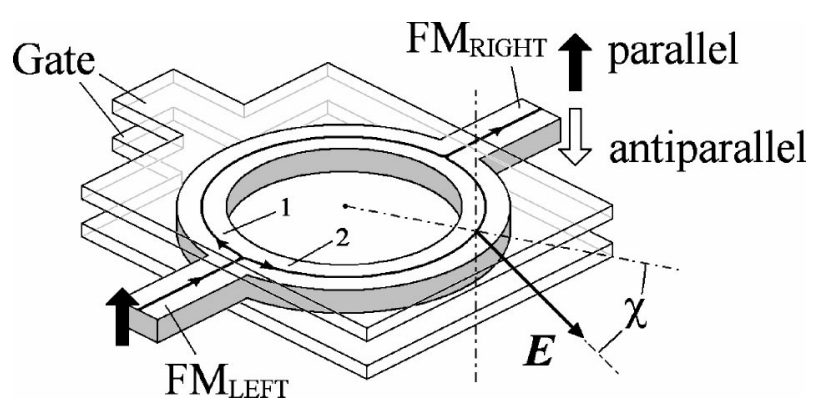

FIG. 1. Schematic picture of the ring geometry with two ferromagnetic filter electrodes in the presence of a texture electric field. The spin carrier travels around the ring with the spin-orbit interaction. The arrows represent the magnetization vectors of electrodes. In the right electrode, the filled (hollow) arrow represents the magnetization vector for the parallel (antiparallel) alignment.

are applied on the ring, which is shown in Fig 1. In this design configuration, it is possible to let only one kind of polarized spin enter and the specific polarized spin, corresponding to a certain polarization switched from parallel $\mathrm{P}$ (ferromagnetic) to antiparallel AP (antiferromagnetic) alignment, and leave the ring through the contacts. When the incoming polarized electron travels along the ring, it suffers the spin flip due to the SO interaction and acquires an AC phase in its wave function. It is worth pointing out that the polarized electrons, whose polarized directions are different from the polarization in ferromagnetic filters, prefer to bypass the intersections between ring and ferromagnetic electrodes rather than to propagate around the ring as a persistent current. As a result of the constraint in polarizations of both incoming and outgoing electrons, the spin precession in the spin conduction is completely determined by the polarized alignments of electrodes. This polarization rotation $(\mathrm{P} \rightarrow \mathrm{AP})$ changes the transport properties of the system. Note that in the ring region, the polarized electrons in the reverse direction of ferromagnetic filter, although not passing the contacts, are still present to travel in the ring. Because SO interaction makes spin flip during their travel on the ring, these carriers with a reverse polarization may be modulated to the polarization parallel to that in the filter by precession and leave the ring through the contact. The output polarized current is sensitive to the spin degree of freedom and its sign can be switched from zero to finite value, depending on the relative magnetization of the ferromagnetic filter electrodes. The interference of polarized electrons makes the polarized conductances to be characterized by geometric phases which originate from the interplay between an electron's spin and orbital degrees of freedom. It makes sense that the spin interference manifests itself as the spin AA phase associated with the spin-polarized states arising from SO interaction. The geometric phases are robust against any decoherence source. Based on this property, the geometric phase control of large magnetoresistance can be anticipated.

The essential ingredient of the ballistic electron transport is the Landauer-Büttiker formula, ${ }^{12}$ which expresses the conductance $G_{\sigma \sigma^{\prime}}$ in the form

$$
G_{\sigma \sigma^{\prime}}=\frac{e^{2}}{h} T_{\sigma \sigma^{\prime}}\left(E_{F}\right),
$$

where $T_{\sigma \sigma^{\prime}}\left(E_{F}\right)$ is a transmission probability for the incident polarized electrons with spin $\sigma(\sigma=\uparrow, \downarrow)$ being transmitted to the outgoing spin state $\sigma^{\prime}\left(\sigma^{\prime}=\uparrow, \downarrow\right)$, and $E_{F}$ is the Fermi energy of electrons in the ferromagnets. In a free-electron approximation of the conduction electrons inside each ferromagnet, the energy of longitudinal motion of polarized electron is equal to $E_{F}$. The resistance is arisen from the electronic scattering by $\mathrm{SO}$ interaction in the ring. In the following calculations we can show that $T_{\sigma \sigma^{\prime}}\left(E_{F}\right)$ is notably filter-alignment-dependent and also depends on the texture electric field. First, we will calculate the transmission probability for the different set up of ferromagnetic filter electrodes, which are switched from parallel $\mathrm{P}$ (ferromagnetic) to antiparallel AP (antiferromagnetic) alignment. We begin with the longitudinally effective one-electron Hamiltonian, including the spin degree of freedom of ferromagnets along the $x$ axis

$$
H_{\text {filter }}=\left(\begin{array}{cc}
\mathbf{p}^{2} / 2 m_{e}+U_{\uparrow}(x) & 0 \\
0 & \mathbf{p}^{2} / 2 m_{e}+U_{\downarrow}(x)
\end{array}\right),
$$

where $m_{e}$ is the effective mass of electron. $U_{\sigma}(x)(\sigma=\uparrow, \downarrow)$ is the constriction potential assumed to allow those electrons with a certain orientation to be transmitted. If the ferromagnetic filter electrodes are switched in a parallel alignment $(\mathrm{P})$, $U_{\uparrow}(x)=0$ and $U_{\downarrow}(x)=\infty$, or $U_{\uparrow}(x)=\infty$ and $U_{\downarrow}(x)=0, x<0$ and $x>2 a$. If the ferromagnetic filter electrodes are switched in an antiparallel alignment (AP), $U_{\uparrow}(x)=0$ and $U_{\downarrow}(x)=\infty$, $x<0$, and $U_{\uparrow}(x)=\infty$ and $U_{\downarrow}(x)=0, x>2 a$; or $U_{\uparrow}(x)=\infty$ and $U_{\downarrow}(x)=0, x<0$, and $U_{\uparrow}(x)=0$ and $U_{\downarrow}(x)=\infty, x>2 a$. Therefore, outside of the left and right boundaries of ring (scattering region), the wave functions of electrons are plane wave with the polarized-spin corresponding to these switched alignment. In brief, we consider an incident plane wave with spin- $\sigma_{i}$ and a unit incident particle flux in the left region. It can be regarded as initial state of spin carrier prepared for being injected and transmitted through the ring.

$$
\begin{gathered}
\Psi_{l}^{\sigma}(x)=e^{i k x}\left|\sigma_{i}\right\rangle+e^{-i k x} r_{P / A P}^{\sigma \sigma^{\prime}}\left|\sigma_{i}^{\prime}\right\rangle, \\
\Psi_{r}^{\sigma}(x)=e^{i k x} t_{P / A P}^{\sigma \sigma^{\prime}}\left|\sigma_{f}^{\prime}\right\rangle,
\end{gathered}
$$

where $t_{P / A P}^{\sigma \sigma^{\prime}}\left(r_{P / A P}^{\sigma \sigma^{\prime}}\right)$ is the transmission (reflection) coefficient, $\left|\sigma_{i}^{\prime}\right\rangle$ and $\left|\sigma_{f}^{\prime}\right\rangle$ label the spin states of incoming and outgoing electrons with $\sigma_{i / f}=\uparrow, \downarrow$ (spin quantized along the $z$ axis). The subscript $P(A P)$ represents a parallel (antiparallel) alignment of ferromagnetic filter electrodes so that $\sigma_{i}=\sigma_{f}$ $\left(\sigma_{i}=\bar{\sigma}_{f}\right)$.

In order to calculate the transmission probabilities $T_{\sigma \sigma^{\prime}}\left(E_{F}\right) \quad\left(=\left|t_{P / A P}^{\sigma \sigma^{\prime}}\right|^{2}\right)$ and to demonstrate the modulation of spin precession as a function of the angle and the strength of texture electric field in the scattering region, we explain how the geometric control parameters are arisen when an electron transmits through the AC ring. We consider a symmetric onedimensional (1D) ballistic mesoscopic ring lying in the $x-y$ 
plane. We assume that the length of the ring, while possibly large in comparison to a typical carrier wavelength, is smaller than the mean-free path and the spin diffusion length $L_{s}$, which is, in turn, smaller than the geometric size of the system. The effective Hamiltonian for the noninteracting electrons in the ring reads

$$
H_{\text {ring }}=\frac{1}{2 m_{e}}\left(\mathbf{p}-\frac{e \hbar}{4 m_{e} c^{2}} \sigma \times \mathbf{E}\right)^{2},
$$

where the electron charge is taken to be $-e, \sigma\left(\sigma_{x}, \sigma_{y}\right.$, and $\sigma_{z}$ ) denote the Pauli matrices, and $\mathbf{p}$ is the momentum of electron. The coupling between electron spin and the electric field is included via the SO interaction term, i.e., $\left(e \hbar / 4 m_{e}^{2} c^{2}\right) \sigma \cdot \mathbf{E} \times \mathbf{p}$. In the cylindrical coordinate system, the cylindrically symmetric electric field $\mathbf{E}$ can be expressed as $\mathbf{E}=E\left(\cos \chi \mathbf{e}_{r}-\sin \chi \mathbf{e}_{z}\right)$. With the help of the transformations $\mathbf{e}_{\mathbf{r}}=\cos \theta \mathbf{e}_{\mathbf{x}}+\sin \theta \mathbf{e}_{\mathbf{y}_{13}}$ and $\mathbf{e}_{\theta}=-\sin \theta \mathbf{e}_{\mathbf{x}}+\cos \theta \mathbf{e}_{\mathbf{y}}$, the Hamiltonian (5) becomes ${ }^{13-16}$

$$
H_{\text {ring }}=\frac{\hbar^{2}}{2 m_{e} a^{2}}\left[-i \frac{\partial}{\partial \theta}+\alpha\left(\sin \chi \sigma_{r}+\cos \chi \sigma_{z}\right)\right]^{2}
$$

with $\sigma_{r}=\sigma_{x} \cos \theta+\sigma_{y} \sin \theta$ and $\alpha=-e a E / 4 m_{e} c^{2}$, where $a$ is the radius of ring and $\theta$ is the angular coordinate. For a ballistic 1D ring the eigenstates of Hamiltonian (6) can be obtained analytically as $\psi_{\tau}(\theta)=(1 / \sqrt{2 \pi}) e^{i n \theta}|\tau\rangle$, in which the exponent factor describes the motion along the ring and $|\tau\rangle$ refers to the spin states $\tau= \pm$,

$$
|+\rangle=\left(\begin{array}{c}
\cos \beta / 2 \\
e^{i \theta} \sin \beta / 2
\end{array}\right) \text { and }|-\rangle=\left(\begin{array}{c}
\sin \beta / 2 \\
-e^{i \theta} \cos \beta / 2
\end{array}\right),
$$

where $\beta$ is given by

$$
\tan \beta=\frac{2 \alpha \sin \chi}{2 \alpha \cos \chi+1} .
$$

For clarity, we consider the nonadiabatic geometric evolution of eigenstates $\psi_{\tau}(\theta)$ with $\tau= \pm$. From the theory of the nonadiabatic Aharonov-Casher phase, the quantum mechanical wave function $\psi_{\tau}(\theta)$ accumulates a phase, $\boldsymbol{\Phi}_{A C}^{(\tau)}$, upon a cyclic evolution when the electron undergoes a nonadiabatic evolution along a closed curve in the presence of an orientationally inhomogeneous electric field. The origin of the phase is so-called the AC effect, and the phase can been written as a sum of its geometric phase $\boldsymbol{\Phi}_{A A}^{(\tau)}$ and dynamic SO phase $\boldsymbol{\Phi}_{S O}^{(\tau)}$, i.e., $\boldsymbol{\Phi}_{A C}^{(\tau)}=\boldsymbol{\Phi}_{A A}^{(\tau)}+\boldsymbol{\Phi}_{S O}^{(\tau)}$, which are given by

$$
\boldsymbol{\Phi}_{A A}^{(\tau)}=-\pi(1-\tau \cos \beta) \text { and } \boldsymbol{\Phi}_{S O}^{(\tau)}=-\tau \pi 2 \alpha \cos (\beta-\chi),
$$

respectively. The geometric and dynamic phases associated with the cyclic evolution of states can thereby be identified for all the energy eigenstates to determine the whole energy spectrum

$$
\begin{aligned}
\frac{2 E_{n, \tau}}{\hbar \omega_{0}}= & \left(n-\frac{1}{2 \pi} \boldsymbol{\Phi}_{A C}^{(\tau)}\right)^{2}-\frac{1}{2 \pi} \boldsymbol{\Phi}_{A C}^{(\tau)}\left(1+\frac{1}{2 \pi} \boldsymbol{\Phi}_{A C}^{(\tau)}\right) \\
& +\alpha(\alpha-\cos \chi),
\end{aligned}
$$

where $\omega_{0}=\hbar / m_{e} a^{2}$. In this expression, the first two terms are the orbital and spin energies, and the third term is the zeropoint energy. ${ }^{14}$

Now let us return the implement that the ring is connected to two ferromagnetic filter-electrodes. For convenience, we use the local coordinate system in the circuit such that the coordinate $x$ is taken along the direction of electron current. The origin of the local coordinate is taken at the left intersection. In fact, the choice of the coordinate origin is trivial because it differs by only a phase factor on the transmission amplitude. We label the upper and lower arms of ring with the subscripts 1 and 2, respectively (see Fig. 1). Once the spin-up electrons transmit into the ring through one of the contacts from electrodes, they are transmitted along two arms to arrive the contact at the another end. We expand the incoming state (of a spin-up electron) in terms of the eigenstates of $H_{\text {ring. }}$. Because of a spin-up incident plane wave having a unit incident particle flux in left region, the part for the spin degree of freedom of the state can be in their superposition states: $|0\rangle=\cos (\beta / 2)|+\rangle+\sin (\beta / 2)|-\rangle$. So the wave function of electrons in the ring will be evolved by means of $|\tau\rangle(\tau= \pm)$. The states $|\tau\rangle(\tau= \pm)$ pick up a phase $\boldsymbol{\Phi}_{A C}^{(\tau)} / 2$, when electrons from one of the intersections move to another intersection along one arm in the clockwise direction while picking up a phase $-\boldsymbol{\Phi}_{A C}^{(\tau)} / 2$ if they move in the counter-clockwise direction along another arm. The total phase changes around the whole loop is $\boldsymbol{\Phi}_{A C}^{ \pm}$as expected. In the ring, the electric field may change the momenta of electrons into the same energy as in the ferromagnets in two different spin eigenstates $| \pm\rangle, k_{1}^{ \pm}=k+\boldsymbol{\Phi}_{A C}^{ \pm} / 2 \pi a$ and $k_{2}^{ \pm}=k-\boldsymbol{\Phi}_{A C}^{ \pm} / 2 \pi a$. Therefore, in the $\mathrm{AC}$ ring, the wave functions have the general form of a plane wave with the modified wave vectors for electrons on the arm $\alpha$,

$$
\Psi_{\alpha}^{P / A P}(x)=\sum_{\tau= \pm}\left(C_{\alpha, \tau}^{P / A P} e^{i k_{\alpha}^{(\tau)} x}+D_{\alpha, \tau}^{P / A P} e^{-i k_{\bar{\alpha}}^{(\tau)} x}\right)|\tau\rangle,
$$

( $\alpha=1$ and 2), where $\bar{\alpha}$ indicates that it takes 2 (1) if $\alpha=1(2)$. In this expression we have rewritten the azimuthal angle $\theta$ in the spin state $| \pm\rangle$ in terms of the coordinate $x$ along the direction of electron current. To solve the Schrödinger equation, one must find the unknowns $r_{\sigma \sigma^{\prime}}^{P / A P}, C_{\alpha, \tau}^{P / A P}, D_{\alpha, \tau}^{P / A P}$, and $t_{\sigma \sigma^{\prime}}^{P / A P}$ for the parallel alignment $\sigma=\sigma^{\prime}(=\uparrow, \downarrow)$ and for the antiparallel alignment $\overline{\sigma^{\prime}}=\sigma(=\uparrow, \downarrow)$ by matching the boundary conditions of the wave functions (3) and (10), and their derivatives at the left and right intersections ( $x=0$ and $\pi a$ ). They are the Griffith's boundary conditions at each intersection, in which the corresponding polarized wave function is continuous and the corresponding current component density is conserved. ${ }^{17}$ In our consideration, we assume that the electrons, once injected, have a negligible probability to lose their spin information by escaping into the left electrode. More explicitly, in the left 
intersection $x=0$, the continuity of the wave functions and the current conservation lead

$$
\begin{gathered}
\left.\Psi_{l}^{\sigma}(x)\right|_{x=0}=\left.\boldsymbol{\Psi}_{1 \sigma}(x)\right|_{x=0}=\left.\boldsymbol{\Psi}_{2 \sigma}(x)\right|_{x=0}, \\
\left.\Psi_{1 \bar{\sigma}}(x)\right|_{x=0}=\left.\Psi_{2 \bar{\sigma}}(x)\right|_{x=0}, \\
\left.\frac{\partial \boldsymbol{\Psi}_{l}^{\sigma}(x)}{\partial x}\right|_{x=0}=\left.\frac{\partial \boldsymbol{\Psi}_{1 \sigma}(x)}{\partial x}\right|_{x=0}+\left.\frac{\partial \boldsymbol{\Psi}_{2 \sigma}(x)}{\partial x}\right|_{x=0}, \\
0=\left.\frac{\partial \boldsymbol{\Psi}_{1 \bar{\sigma}}(x)}{\partial x}\right|_{x=0}+\left.\frac{\partial \boldsymbol{\Psi}_{2 \bar{\sigma}}(x)}{\partial(x)}\right|_{x=0}
\end{gathered}
$$

because only spin polarized $\sigma$ electrons in the left filter. In the right intersection $x=\pi a$, the continuity of the wave functions and the current conservation give the boundary conditions as

$$
\begin{gathered}
\left.\Psi_{r}^{\sigma^{\prime}}(x)\right|_{x=\pi a}=\left.\boldsymbol{\Psi}_{1 \sigma^{\prime}}(x)\right|_{x=\pi a}=\left.\boldsymbol{\Psi}_{2 \sigma^{\prime}}(x)\right|_{x=\pi a}, \\
\left.\frac{\partial \boldsymbol{\Psi}_{r}^{\sigma^{\prime}}(x)}{\partial x}\right|_{x=\pi a}=\left.\frac{\partial \boldsymbol{\Psi}_{1 \sigma^{\prime}}(x)}{\partial x}\right|_{x=\pi a}=\left.\boldsymbol{\Psi}_{2 \bar{\sigma}^{\prime}}(x)\right|_{x=\pi a}, \\
0=\frac{\partial \boldsymbol{\Psi}_{1 \bar{\sigma}^{\prime}}(x)}{\partial x}+\left.\frac{\partial \boldsymbol{\Psi}_{2 \sigma^{\prime}}(x)}{\partial x}\right|_{x=\pi a}, \\
\partial(x)
\end{gathered}
$$

because only spin polarized $\sigma^{\prime}$ electrons flow outward the right filter, where we have chosen the derivatives toward the positive direction along the coordinate axis of $x$ in the boundary conditions (11) and (12); $\sigma=\sigma^{\prime}=\uparrow$ or $\downarrow$ corresponds to the parallel magnetizations in filters while $\overline{\sigma^{\prime}}=\sigma=\uparrow$ or $\downarrow$ corresponds to the antiparallel magnetizations in filters. For the parallel (P) magnetic configuration, i.e., the orientations of the magnetic filters of the left and right ferromagnetic electrodes are parallel; the spin transmission coefficients $t_{P}^{\sigma}$ are determined by the boundary conditions (11) with incoming and outgoing electrons with the polarization $\sigma(\sigma=\uparrow$ or $\downarrow)$. In this case those electrons in the spin state $\bar{\sigma}$, which precesses from the spin state $\sigma$, would remain and evolve in the ring. They leave the ring until that they precess to the same spin state as the filters. Only those electrons whose spin polarization is parallel to the magnetization of right filter are transmitted through the whole system. For the antiparallel (AP) magnetic configuration, i.e., the orientations of the magnetic filters of the left and right ferromagnetic electrodes are antiparallel, those electrons in the same spin state as the incoming electron on the right intersection would evolve in the ring. Differing from those in parallel (P) magnetic configuration, only the incoming electrons whose spin polarization is completely flipped in the right intersection are transmitted through the contact to the right electrode. During the flight time from injector to detector, the spin direction of electrons can therefore only be altered by coherent precession through SO interaction in the presence of an external electric field. Then, the spin-polarized tunnelling conductance depends on the relative phase between the magnetizations of two ferromagnetic electrodes, the applied electric field, which produces an $\mathrm{AC}$ phase to make the spin precession on the ring when polarized electrons move alone the ring with SO interaction, the bias voltage, and the temperature.

The transmission coefficients of an incoming electron with spin $\sigma$ for the parallel (P) and antiparallel (AP) alignments are given by

$$
t_{P / A P}^{\sigma}=\left|t_{P / A P}^{\sigma}\right| e^{i \Theta_{P / A P}^{\sigma},}
$$

where $\left|t_{P / A P}^{\sigma}\right|=\Gamma_{P / A P}^{\sigma}\left(M_{P / A P}^{2}+N_{P / A P}^{2}\right)^{-1 / 2}$ and $\tan \Theta_{P / A P}$ $=M_{P / A P} / N_{P / A P}$. It is found that

$$
\begin{gathered}
\Gamma_{P}^{\sigma}=\sin k a \pi \cos \beta \sin \frac{\Delta}{2}\left(\sin ^{2} \frac{\Delta}{2}-\cos ^{2} k a \pi\right), \\
\Gamma_{A P}^{\sigma}=\sin k a \pi \sin \beta \sin \frac{\Delta}{2}\left(\sin ^{2} \frac{\Delta}{2}-\cos ^{2} k a \pi\right), \\
M_{P}=\left(\sin ^{2} \frac{\Delta}{2}-\cos ^{2} k a \pi\right)^{2}+\frac{1}{4} \sin ^{2} k a \pi\left(\cos ^{2} \beta \sin ^{2} \frac{\Delta}{2}\right. \\
\left.-\cos ^{2} k a \pi\right), \\
M_{A P}=\left(\sin ^{2} \frac{\Delta}{2}-\cos ^{2} k a \pi\right)^{2}+\frac{1}{4} \sin ^{2} k a \pi\left(\sin ^{2} \beta \sin ^{2} \frac{\Delta}{2}\right. \\
\left.\quad-\cos ^{2} k a \pi\right), \\
N_{P}=N_{A P}=\sin k a \pi \cos k a \pi\left(\sin ^{2} \frac{\Delta}{2}-\cos ^{2} k a \pi\right), \\
\quad \text { and } \Delta=\pi \sqrt{(\alpha \sin \chi)^{2}+(1+\alpha \cos \chi)^{2} .}
\end{gathered}
$$

Figure 2 shows the transmission of a polarized electron through the $\mathrm{AC}$ ring. We choose the typical values of the material parameters for ferromagnetic metals of $\mathrm{Ni}, \mathrm{Co}$, and Fe [16]; the Fermi energy is taken to be $E_{F}=3.8 \mathrm{eV}$ $\left(k_{F}=1.0 \AA^{-1}\right)$. The circumference of the circuit is taken to be $2 \pi a=200 \AA(a \sim 30 \AA)$ about $50-100$ atoms in size. We inject the spin-up polarized electrons into the ring and measure the output flux of spin-up (parallel filter-electrode alignment) and spin-down (antiparallel filter-electrode alignment) electrons. In Fig. 2, the curves for the double polarizations in electrodes are drawn for comparison. In comparison with the transmission of ferromagnetic electrodes (no-filters), we notice that these oscillations are not in the same structure as those with two possible outgoing polarized electrons. The oscillations exhibit an extra structure that depends on the range of electric field. Sweeping the electric field from negative to positive, the transmission can be modulated more efficiently: we can flip the spin of the incident electrons or block it completely when the magnetization of device switches from a parallel to antiparallel configurations, and thus, a spin transistor is established. The transmission is periodic with respect to the electric field. Also found is a 


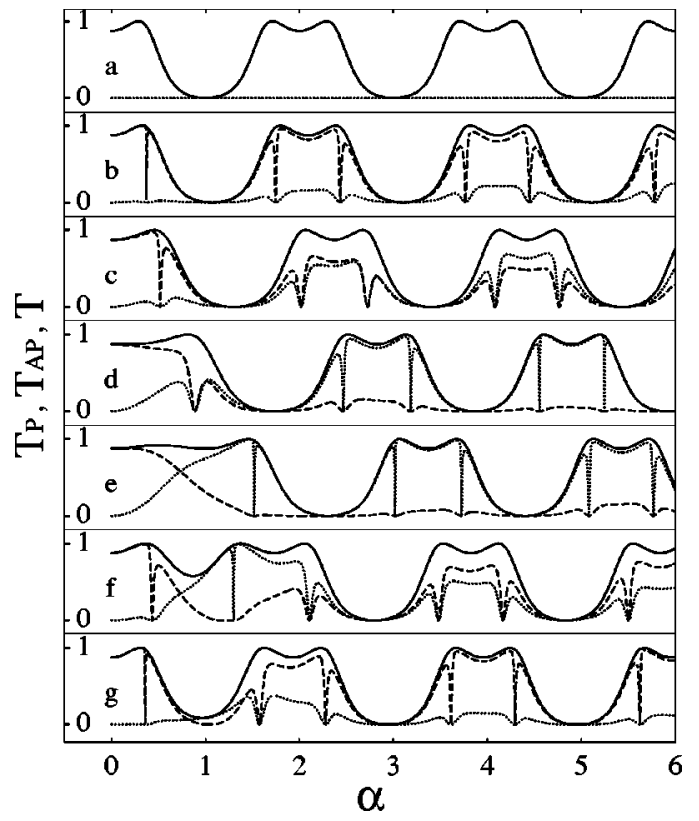

FIG. 2. The transmission coefficients $\left(T_{P / A P}\right)$ vs. the applied electric field (in the unit $e a / 4 m_{e} c^{2}$ ) for several values of texture angle $\chi(0, \pi / 6, \pi / 3, \pi / 2,2 \pi / 3,5 \pi / 6$, and $11 \pi / 12)$. Dashed line is for parallel alignment $T_{P}$ and dotted line is for antiparallel alignment $T_{A P}$. The solid line corresponds to the case with double polarization in the ferromagnets $T$.

square-type transmission curve with a shape dip, which has been found for the modulation of spin-current in the structure of periodically stubbed electron waveguides, ${ }^{18}$ but with a narrow gap. Differing from the cases with changing the width of the waveguide and the asymmetry parameter, the transmissions here are the function of electrical field $E$ and the tilt angle $\chi$. Looking at the curves $T_{P}$ and $T_{A P}$ in Fig. 2, we notice that they are quite different from each other. We found the followings: (1) both $T_{P}$ and $T_{A P}$ oscillate with the same periodic rate as that of double polarizations in outgoing electron, although the amplitudes of the square-type transmission curves are different; (2) the amplitudes of the square-type transmission curves decay with increase of strength of electric field for parallel alignment while that enhances with the increase of the electric field for antiparallel alignment, as a sequence of the spin-flip transmission that contributes to $T_{A P}$ with increasing Rashba coupling. It is found that the spin-flip is blocked in the case of $\chi=0$. It is known that the spin has not precessed when the electric field is along the radial direction, where the AA phase disappears. Because no spin current is modulated when the AA phase vanishes, the spin polarizability remains unchanged. So the transmissions for the antiparallel polarization filters are completely blocked. With increase of the tilt angle $\chi$, the AA phase is presented. The transmissions for the parallel polarization filters are decreased when the electric field is increased, which is equivalent to an increase in the SO interaction, while the transmissions for antiparallel polarization filters are increased. This is an explicit signal of spin-flip. At $\chi=\pi$, it is again that the spin-flip is blocked. It is noted that $\chi=\pi$ is a mirror point.

From plots of transmissions in Fig. 2, it is observed

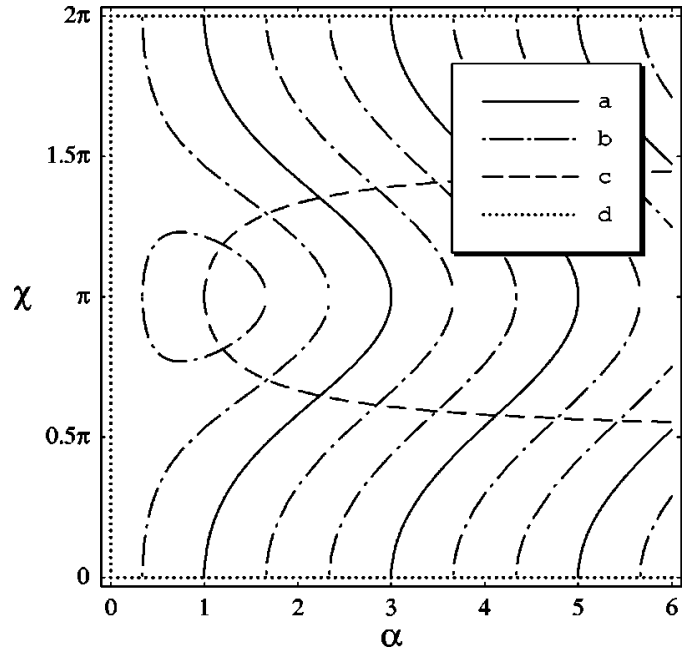

FIG. 3. A section of the transmission coefficients $\left(T_{P / A P}=0\right)$ for the completely blocked incident electrons. A parallel configuration: (a), (b), and (c); while an antiparallel configuration: (a), (b), (d), where (a) $\sin \Delta / 2=0$, (b) $\sin ^{2} \Delta / 2-\cos ^{2} k a \pi=0$, (c) $\cos \beta=0$, and (d) $\sin \beta=0$.

that there are the extra structures in the square-type transmission curve. It is found that near of the edges of the squaretype transmission curves there are the peaks accompanied to the main curves. Between them there appears the blocking of transmissions at appropriate values of electric field. It is found that the small change of electric field near of these edges the transmissions start to increase steeply. Such kind of the extra structure have also been observed in the semiconductor heterostructures. ${ }^{18}$ To demonstrate the extra structure corresponding to blocked transmission via a manifestation of Berry's phase in a system with SO interaction, we cut the section of $T_{P / A P}=0$. In Fig. 3 we have shown the completely blocked incident electrons for both the parallel to antiparallel configurations for appropriate $\alpha$ and $\chi$. Blocking transmission in parallel alignment is at $\cos \beta$ $=0$ (dashed line), $\sin \Delta / 2=0$ (solid line), and $\sin ^{2} \Delta / 2$ $-\cos ^{2} k a \pi=0$ (dashed-dot line), while it is at $\sin \beta=0$ (dotted line), $\sin \Delta / 2=0$, and $\sin ^{2} \Delta / 2-\cos ^{2} k a \pi=0$ for antiparallel alignment.

In Fig. 4, we plot the magnetoconductance ratio, which is given by the relative change $\Delta G / G_{a v}=\left(T_{P}-T_{A P}\right) /\left(T_{P}+T_{A P}\right)$. The magnetoconductance ratio in Fig. 4 can be controlled via a cylindrically symmetric electric field. The oscillating magnetoconductance is observed due to spin precession. This feature is equivalent to the spin-field-effect transistor proposed by Datta and Das. ${ }^{1}$ In comparison with the magnetoconductance ratio in the general ferromagnetic electrodes, ${ }^{9}$ we found the distinct differences in the presence of the filter electrodes. The filters provide intrinsic AA phase-dependent peaks that give rise to the extra oscillating effect in magnetoconductance. These extra structures correspond to the blocking points in the transmissions (see Fig. 5), i.e., $\cos \beta=0, \quad \sin \beta=0, \quad$ and $\sin ^{2} \Delta / 2$ $-\cos ^{2} k a \pi=0$.

In the remainder of the paper, we comment on the possibility for measurement of AA phase in the present spin-filter system. Mathur and Stone pointed out that the observable 


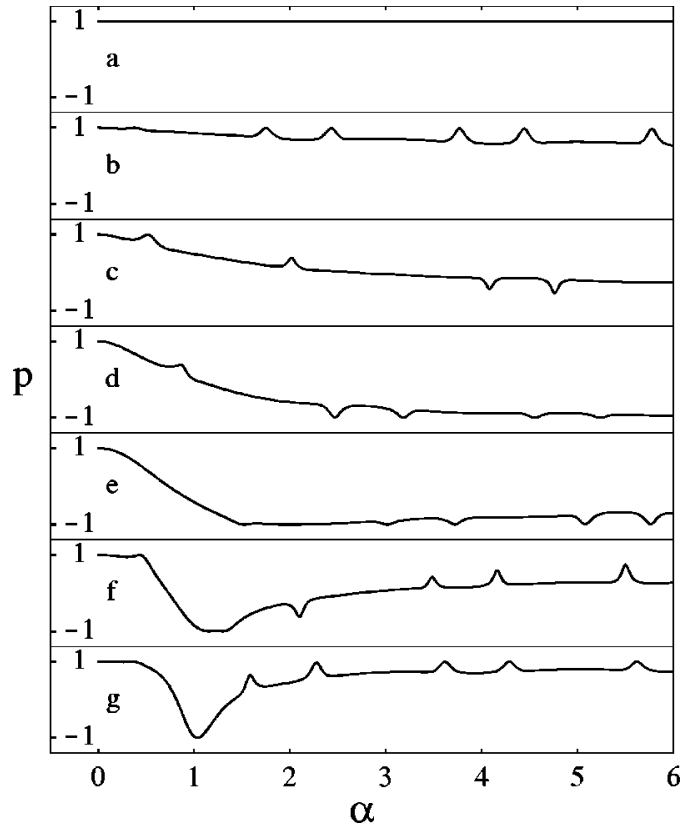

FIG. 4. The magnetoconductance ratio, $\mathrm{p}=\Delta G / G_{a v}$, as the function of electric field in the unit $e a / 4 m_{e} c^{2}$ and its angle of texture $\chi$ $(0, \pi / 6, \pi / 3, \pi / 2,2 \pi / 3,5 \pi / 6$, and $11 \pi / 12)$.

phenomena induced by SO interactions are essentially the manifestation of the $\mathrm{AC}$ effect and proposed an experiment to observe the AC oscillation of the conductance in semiconductor samples. ${ }^{19,20}$ In the above description, the geometric manipulation of electron spin in a mesoscopic ring by the electrical approach and detection of the geometric phase have been discussed. The device can be regarded as a geometric spintronic Faraday rotation spectroscopy, which is used to describe the phenomenon of the polarization of a polarized electron, after it has been transmitted through a system being precessed around the electron propagation or the wave-vector direction. In order to show the geometric manipulation of electron spin and the detection of the geo-

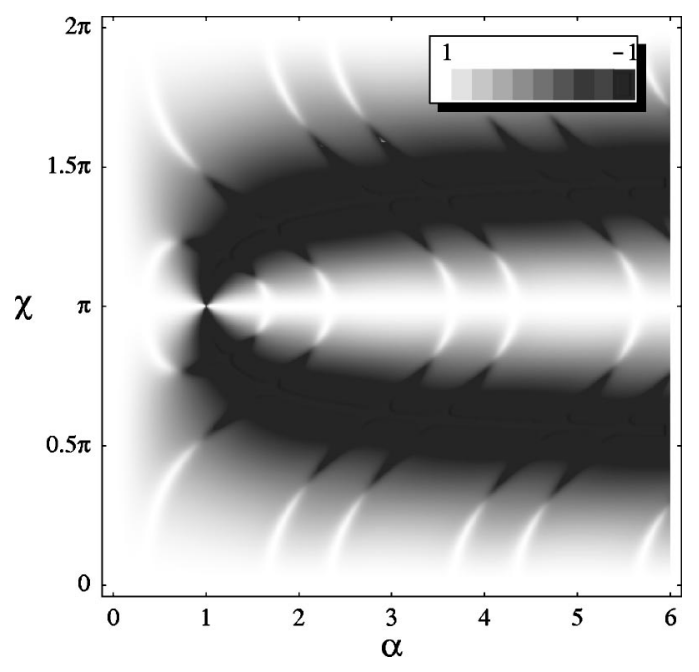

FIG. 5. The contour plot for the magnetoconductance ratio, $\Delta G / G_{a v}$, as the function of electric field in the unit $e a / 4 m_{e} c^{2}$ and its angle of texture $\chi$. metric phase via the extremely sensitive geometric spintronic Faraday rotation spectroscopy, we discuss the spin motion acquiring a geometric phase that, in turn, generates an observable effect in spin transport in filter. We check the consequence of the spin precession on the eigenstates of $\sigma_{z}$, $|0\rangle=\cos \beta / 2|+\rangle+\sin \beta / 2|-\rangle \quad$ and $\quad|1\rangle=\sin \beta / 2|+\rangle$ $-\cos \beta / 2|-\rangle$. When an electron processes the evolution from one intersection to another in the ring, the state $|+\rangle$ acquires a phase $e^{i \Phi_{A C}^{(+)}} / 2$ and the state $|-\rangle$ acquires a phase $e^{i \Phi_{A C}^{(-)} / 2}$. As the mix states the quantum-state evolutions for $\boldsymbol{\Psi}_{S}^{T}=(|0\rangle,|1\rangle)$ are described by $\boldsymbol{\Psi}_{S} \rightarrow \mathbf{M} \boldsymbol{\Psi}_{S}$, where

$\mathbf{M}=\sin \frac{\boldsymbol{\Phi}_{A C}^{(+)}}{2}\left(\begin{array}{cc}\tan ^{-1} \boldsymbol{\Phi}_{A C}^{(+)} / 2+i \cos \beta & i \sin \beta \\ i \sin \beta & \tan ^{-1} \boldsymbol{\Phi}_{A C}^{(+)} / 2-i \cos \beta\end{array}\right)$.

From these expressions, it is seen that the AC phase plays a role of precessing the spin states $|0\rangle$ and $|1\rangle$ into their superposition states. As a consequence, the geometric modulation of electron polarization is realized. Unfortunately, the phases accumulated in this way have both the geometric and the dynamic contributions. To detect merely the geometric phase via the interferometry, it is necessary to eliminate the dynamic phase. To implement the elimination of the dynamic phase, we can make the polarized electrons evolve twice by suddenly changing the bias between two filters for the antiparallel alignment after the first evolution has been completed. Then the second cyclic evolution of the polarized electrons retraces the first one, but following the reverse path. Taking the precession of the initial state $|0\rangle$ as an example for demonstrating the detection of geometric phase, two steps are described as follows:

$$
\begin{aligned}
|0\rangle & \rightarrow \frac{1}{2} \sin \beta\left(e^{i \boldsymbol{\Phi}_{A A}^{(+)}} e^{-i E^{(+)} t}+e^{i \boldsymbol{\Phi}_{A A}^{(-)}} e^{-i E^{(-)} t}\right)|1\rangle \\
& \rightarrow \frac{i}{2} \sin ^{2} \beta \sin 2 \boldsymbol{\Phi}_{A A}^{(+)} e^{-i\left(E^{(+)}-E^{(-)}\right) t}|0\rangle .
\end{aligned}
$$

Thus we found that the net effect of this compound operation is to cancel the dynamic phase and maintain the geometric one.

The generalization of the geometric phase associated with the evolution of the mixed state is one important problem in a quantum system, which relates to generalize the parallel transport condition. The geometric phase associated with the no-jump trajectory is the same as the one acquired by an isolated system evolving under the same Hamiltonian. In fact, the geometric phase is actually robust against all of the polarizations of filters but spin flip. The final geometric phase is affected by spin flips manifested by the SO interaction during the polarized electron transporting in the ring. From Fig. 2 we have found that in this geometric evolution, i.e., modulating $\chi$ and $E$, the geometric phase associated with the strength of flips during the transport is sensitive for the transport of the spin-polarized electrons. This can be regarded as a simple geometrical explanation for AA phase associated with the spin precession.

In summary, the polarized spin transmissions across an 
AC ring with the parallel and antiparallel magnetized alignments of electrodes have been studied. We have shown that the spin filtering can be fully manipulated with the AC effect. The origin is the spin flip via spin precession, which can easily be controlled via a cylindrically symmetric electric field. There are several advantages to the present investigation. As a precise representation of geometric spintronic Faraday rotation spectroscopy, we present a geometric explanation as to the phenomenon of the polarization of spinpolarized electrons being precessed around the electron propagation or the wave-vector direction during its transmitting through a system. We propose it to be an interference scheme based on the ring setup for detection of the geometric phase.

This research was supported by NNSFC and NSF of Guangdong Province under Grants No. 90103027, No. 10274069, and No. 011151; the Research Grant Council of Hong Kong; and a CRCG grant of the University of Hong Kong.
${ }^{1}$ S. Datta and B. Das, Appl. Phys. Lett. 56, 665 (1990).

${ }^{2}$ G. Salis, Y. Kao, K. Ensslin, D.C. Driscoll, A.C. Gossard, and D.D. Awschalom, Nature (London) 414, 619 (2001).

${ }^{3}$ J.M. Kikkawa and D.D. Awschalom, Nature (London) 397, 139 (1999).

${ }^{4}$ F. Mireles and G. Kirczenow, Phys. Rev. B 64, 024426 (2001); A.V. Moroz and C.H.W. Barnes, ibid. 60, 14272 (1999).

${ }^{5}$ M. Johnson, J. Magn. Magn. Mater. 140-144, 21 (1995).

${ }^{6}$ J.C. Slonczewshi, J. Magn. Magn. Mater. 159, L1 (1996); 195, L261 (1999); L. Berger, Phys. Rev. B 54, 9353 (1996); J. Appl. Phys. 81, 4880 (1997); M. Tsoi, A.G.M. Jansen, J. Bass, W.C. Chiang, M. Seck, V. Tsoi, and P. Wyder, Phys. Rev. Lett. 80, 4281 (1998); 81, 493 (1998); M. Tsoi, A.G. Jansen, J. Bass, W.C. Chiang, V. Tsi, and P. Wyder, Nature (London) 406, 46 (2000); E.B. Myers, D.C. Ralph, J.A. Katine, R.N. Louie, and R.A. Buhrman, Science 285, 867 (1999); J.Z. Sun, J. Magn. Magn. Mater. 202, 157 (1999); J.A. Katine, F.J. Albert, R.A. Buhrman, E.B. Myers, and D.C. Ralph, Phys. Rev. Lett. 84, 3149 (2000); F.J. Albert, J.A. Katine, R.A. Buhrman, and D.C. Ralph, Appl. Phys. Lett. 77, 3809 (2000).

${ }^{7}$ D. Frustaglia, M. Henschel, and K.A. Chao, Phys. Rev. Lett. 87, 256602 (2001).
${ }^{8}$ M. Popp, D. Frustalia, and K. Richter, Nanotechnology 14, 347 (2003).

${ }^{9}$ S.Q. Shen, Z.J. Li, and Z.S. Ma, Appl. Phys. Lett. 84, 996 (2004).

${ }^{10}$ Y. Aharonov and A. Casher, Phys. Rev. Lett. 53, 319 (1984).

${ }^{11}$ Y. Aharonov and J. Anandan, Phys. Rev. Lett. 58, 1593 (1987).

${ }^{12}$ R. Landauer, Philos. Mag. 21, 863 (1997); M. Büttiker, Y. Imry, R. Landauer, and S. Pinhas, Phys. Rev. B 31, 6207 (1985).

${ }^{13}$ A.G. Aronov and Y.B. Lyanda-Geller, Phys. Rev. Lett. 70, 343 (1993).

${ }^{14}$ Y.S. Yi, T.Z. Qian, and Z.B. Su, Phys. Rev. B 55, 10631 (1997).

${ }^{15}$ T. Choi, S.Y. Cho, C.M. Ryu, and C.K. Kim, Phys. Rev. B 56, 4825 (1997).

${ }^{16}$ P.M. Levy and S. Zhang, Phys. Rev. Lett. 79, 5110 (1997); J.F. Gregg et al., ibid. 77, 1580 (1996).

${ }^{17}$ S. Griffith, Trans. Faraday Soc. 49, 345 (1953); S. Griffith, Trans. Faraday Soc. 49, 650 (1953); J. Xia, Phys. Rev. B 45, 3593 (1992); A.M. Jayannavar and P. Singha Deo, ibid. 49, 13685 (1994).

${ }^{18}$ X.F. Wang, P. Vasilopoulos, and F.M. Peeters, Appl. Phys. Lett. 80, 1400 (2002); Phys. Rev. B 65, 165217 (2002).

${ }^{19}$ H. Mathur and A.D. Stone, Phys. Rev. Lett. 68, 2964 (1992).

${ }^{20}$ L. Hu, J. Gao, and S.Q. Shen, Phys. Rev. B 69, 165304 (2004). 